\title{
Performance evaluation of microbead and ELISA assays for follicular G- CSF: a non-invasive biomarker of oocyte developmental competence for embryo implantation"
}

\author{
Nathalie Lédée ${ }^{\mathrm{a}, \mathrm{b}}$, Carine Munaut ${ }^{\mathrm{b}}$, Valérie Sérazin ${ }^{\mathrm{c}}$, Sophie Perrier d'Hauterive ${ }^{\mathrm{b}}$, Letizia Lombardellid ${ }^{\mathrm{d}}$, Federica \\ Logiodice $^{\mathrm{d}}$, Robert Wainer ${ }^{\mathrm{c}}$, Virginie Gridelet ${ }^{\mathrm{b}}$, Gérard Chaouat ${ }^{\mathrm{a}}$, Francis Frankenne ${ }^{\mathrm{b}}$, Jean Michel Foidart ${ }^{\mathrm{b}}$, \\ Marie-Pierre Piccinni ${ }^{\mathrm{d}}$ \\ a INSERM U782, Université Paris-Sud, UMR-S0782, Clamart F-92140, France \\ ${ }^{\mathrm{b}}$ Université de Liège, Département universitaire de Gynécologie et d'obstétrique, CHR la Citadelle, Liège, Belgium \\ ' Service de Médecine de la Reproduction et de Biologie Moléculaire, CHI Poissy St. Germain, EA-2493 Poissy, France \\ ${ }^{\mathrm{d}}$ Centre of Excellence for Research, Transfer and High Education DENOTHE of the University of Florence, Department of Internal \\ Medicine-Immuno-aiiergoiogy Unit, viale Morgagni 85, 50134 Florence, Italy
}

\begin{abstract}
G-CSF in individual follicular fluids correlates with the potential of the corresponding embryo to result in a live birth after transfer in IVF. To evaluate the requirements for routine follicular fluid G-CSF quantification, we compared follicular fluid G-CSF measurements made with two multiplexed microbead assays purchased from Bio-Rad Laboratories and R\&D Systems, and a commercial G-CSF ELISA (R\&D Systems). Individual follicular fluids $(n=139)$ associated with transferred embryos were analysed to determine cytokine profile and the fate of each transferred embryo was recorded. The effect of multiplexing as well as comparison of the respective performances of the microbead assay with a flow cytometry assay was explored. Multivariable logistic regression analysis was performed and receiver operating characteristic (ROC) analysis was used to determine the performance and sensitivity/specificity of each method for individual follicular fluids. Covariate factors known to influence IVF outcome such as age, serum oestradiol and embryo score were systematically integrated in each analysis. The quantification of follicular fluid G-CSF using microbead assay methodologies, but not ELISA, yielded results showing the utility of follicular fluid G-CSF as a biomarker predictive of a successful delivery $\left(\mathrm{Au}_{\mathrm{roc}}: 0.77[0.68-0.84](p=0.003)\right.$ and 0.75 [0.66-0.82] $(p=0.004)$ for Bio-Rad and R\&D Systems microbead assays respectively), whereas follicular fluid G-CSF values quantified by ELISA were not predictive $\left(\mathrm{Au}_{\mathrm{roc}}: 0.61[0.52-0.70] p=0.84\right)$. Microbead assay and flow cytometry appeared similarly efficient for quantifying follicular fluid G-CSF and multiplex versus single-plex assays did not influence the reliability of quantification.
\end{abstract}

Keywords : Follicular fluid ; G-CSF ; Implantation ; Pregnancy ; Bead-based assay ; IVF/ICSI

\section{Introduction}

In previous studies, we simultaneously measured with a multiplex bead-based immunoassay 27 cytokines and chemokines in each follicular fluid collected from individual follicles after either ovarian hyperstimulation or monitored natural cycles. Each follicular fluid analysed was the source of an oocyte subsequently fertilized and transferred (Lédée et al, 2008, 2010). We demonstrated that the level of granulocyte colony-stimulating factor (G-CSF) in individual follicular fluid samples was correlated with the birth potential of the corresponding embryo. Studies using Western blotting and immunochemistry techniques have identified G-CSF protein and its receptor in the ovary, mainly in the granulosa cells of the follicles, and found that it surges at ovulation (Salmassi et al., 2004; Yanagi et al., 2002). Salmassi et al. (2004) reported an increase in G-CSF levels 10 days after ovulation in the serum of successful hyperstimulated and natural IVF/ICSI cycles (Salmassi et al., 2005).

\footnotetext{
* Financial support: This work was supported by the European network of excellence EMBIC (contract 512040) and the french agency of biomedicine.
} 
Assessing the full potential of each individual oocyte or embryo to develop and successfully implant is desirable in assisted reproduction. It is the key to decreasing the mortality and morbidity generated by multiple pregnancies and also the adverse psychological effects of explained negative results. During ART cycles, only approximately 5\% of fresh oocytes result in a baby (Patrizio and Sakkas, 2009). Morphological parameters for non-invasive quality embryo assessment have been developed, although their biological relevance has recently been re-evaluated (Guerif et al., 2007).

In determining the utility of G-CSF as a biomarker for oocyte developmental competence and implantation potential, the choice of the technology as well as the validation of standard operating procedures is likely to be important. The major question remaining before routine application in IVF programs is the nature of the most appropriate technique for detecting G-CSF in follicular fluid, in order to reliably predict the implantation potential of the resulting embryo.

\section{Materials and methods}

\subsection{Study protocol}

\subsubsection{Patients}

From September 2005 through March 2007, we prospectively recruited 84 women undergoing their first attempt of fertility treatment by intracytoplasmic sperm injection (ICSI). Each patient was included only once. The choice to only include ICSI patients was related to the treatment protocol, which aimed to define the cytokine content of individual follicular fluids in morphologically mature oocytes at the time of collection. All patients gave fully informed consent, and the Institutional Review Board (Comité Consultatif de Protection des Personnes, Poissy-St Germain en Laye) approved this investigation.

\subsubsection{Treatments}

Ovarian hyperstimulation protocols were selected by each treating physician. The response to the stimulation was monitored by serial blood tests together with ultrasound assessment of follicular and endometrial growth. Ovulation was triggered when at least 4 follicles had reached $16 \mathrm{~mm}$. We classified the responses to ovarian stimulation into three categories: (1 ) low response; serum oestradiol below $1500 \mathrm{pg} / \mathrm{ml}$ on the day ovulation was triggered, (2) moderate responders; serum oestradiol between 1500 and $3500 \mathrm{pg} / \mathrm{ml}$, and (3) high responders; serum oestradiol over $3500 \mathrm{pg} / \mathrm{ml}$.

The oocytes were retrieved by aspiration 35-36 $\mathrm{h}$ after triggering ovulation, using general or local anaesthesia and vaginal ultrasound guidance, with the aid of an individual $10 \mathrm{ml}$ syringe for each follicle as previously described (Lédée et al., 2008). Oocytes were collected individually and the cumulus and corona cells were removed by incubation in 80 IU hyaluronidase (Fertipro, JCD, France). The oocytes were injected with a single sperm in a $5 \mu \mathrm{l}$ droplet of Ferticult Hepes (JCD, France) under standard conditions, and individually cultured in a $40 \mu \mathrm{l}$ microdroplet of ISM1 (Medicult, France) under oil at $37^{\circ} \mathrm{C}$ in a $5 \% \mathrm{CO}_{2}$ humidified atmosphere. On day 2 , the embryos were analysed to measure (a) blastomere fragmentation (grade 1: 10\% or less; grade 2, 10-30\%; grade 3, 30-50\%; grade 4, over 50\%, and the grade was marked down when blastomeres were unequal in size) and (b) number of blastomeres. An embryo score, calculated as the number of blastomeres $\times$ (5 - grade), was attributed to each embryo (Steer et al., 1992). The optimal category was defined as those with 4-5 cells on day 2 , $10 \%$ or less of fragmentation and equal blastomere size.

\subsubsection{Follicular fluid samples}

The presence of an oocyte in each follicular fluid sample was assessed immediately, and those samples without an oocyte were discarded. The samples were initially stored at $-20^{\circ} \mathrm{C}$ and then at $-80^{\circ} \mathrm{C}$ until they were assayed. Only those follicular fluid samples corresponding to embryos transferred on day 2 were analysed. From 162 embryos transferred on day 2, 139 follicular fluids were analysed. Each follicular fluid sample was blinded, identified only by patient number, stimulation number, and oocyte number within the cohort. Attaching names (and therefore results) to the numbers required a key not available to those performing the test (Medifirst SA, Guyancourt, France). Follicular fluids from $n=139$ follicles were thawed and analysed, without any other manipulation or dilution, by two multiplexed microbead assays purchased from two different companies (R\&D Systems, Minneapolis, MN, USA and Bio-Rad Laboratories, Hercules, CA, USA), as well as by a standard commercial solid-phase ELISA for G-CSF (Quantikine G-CSF kit, R\&D Systems, Wresbaden, Germany). 


\subsection{Methods used to quantify cytokines in follicular fluids}

Both multiplexed microbead assays were tested to simultaneously measure multiple analytes in individual follicular fluids by flow cytometric resolution of spectrally distinct microspheres coupled with capture molecules and reporter fluorochromes bound to detection antibodies. A Luminex system (Luminex Map Technology) was used to read concentrations of cytokines, chemokines and growth factors. The multiplexed microbead assay from Biorad detected IL-1 $\alpha$, IL-1R $\alpha$, IL-2, IL-4, IL-5, IL-6, IL-8, IL-9, IL-10, IL-12, IL-13, IL-15, IL-17, IFN $\alpha$ TNF $\alpha$ G-CSF, GM-CSF, VEGF, PDGF, FGF, IP-10, MCP-1, CCL5, eotaxin, MIP-1 $\alpha$ and MIP-1 $\beta$. A single-plex microbead assay (also purchased from Bio-Rad ${ }^{\circledR}$ ) measured G-CSF alone. The multiplexed microbead assay from R\&D Systems ${ }^{\circledR}$ measured IL-1 $\alpha$, IL-1 $\beta, \Gamma L-1 R \alpha$, IL-2, IL-4, IL-5, IL-6, IL-8, IL-10, IL-17, TNF $\alpha$, IFN7, G-CSF, GM-CSF, VEGF, MIP-1 $\alpha$, MIP- $\beta$, RANTES and MCP-1. Each assay was performed according to the manufacturer instructions.

The limits of detection for follicular fluid G-CSF (defined as mean \pm 2 SD of blank samples, where G-CSF was not present) were $5.3 \pm 8 \mathrm{pg} / \mathrm{ml}$ (range $=0-13 \mathrm{pg} / \mathrm{ml}$ ) for the Bio-Rad multiplexed microbead assay and $3.5 \pm 3$ $\mathrm{pg} / \mathrm{ml}$ (range $=0.5-6.5 \mathrm{pg} / \mathrm{ml}$ ) for the R\&D Systems multiplexed microbead assay. The \% CV for the Bio-Rad multiplex bead-based assay and for the R\&D Systems multiplex bead-based assay is $<5 \%$ for intra-assay variation and $<10 \%$ for interassay variation. In the single-plex microbead assay, the lower limit of G-CSF detection was $1.7 \mathrm{pg} / \mathrm{ml}$.

The G-CSF content of individual follicular fluids was also assayed in the commercial ELISA according to the manufacturer instructions. All samples, standards and controls were assayed in duplicate. Precision was $<5 \%$ for intra-assay variation and $<10 \%$ for inter-assay variation with a detection limit of $20 \mathrm{pg} / \mathrm{ml}$.

For the flow cytometric assay, levels of G-CSF were assayed using the Cytometric Bead Array Human Soluble Protein Flex set (BD Biosciences, cat \#558-264) according to the manufacturer instructions. Flow cytometry analysis was performed using a FACSCanto II (BD Biosciences) and data were analysed with FCAP (BD Biosciences) software. The detection limit was $1.6 \mathrm{pg} / \mathrm{ml}$ and precision was $<3 \%$ for intra-assay variation and $<9 \%$ for inter-assay variation.

\subsection{Statistical analysis}

For the study cohort a mean of 2 embryos were replaced. Thus we were only able to define for each sample its corresponding implantation rate. Implantation rate is defined as the number of intrauterine gestational sacs observed by ultrasound at seven weeks of amenorrhea, expressed as a ratio of the number of transferred embryos. The delivery rate was defined as the ratio of the number of babies born to the number of embryos transferred. Embryos transferred (and their corresponding follicular fluids) were classified according to three categories : embryos which did not implant $(n=104)$, embryos which definitely implanted $(n=13)(2$ embryos replaced resulting in twin fetuses, or 1 embryo replaced resulting in a singleton fetus) and a third category in which a probability of implantation was defined $(n=22)$ (for example, 2 embryos replaced resulting in a singleton fetus). To identify predictive factors of subsequent birth, multivariable logistic regression analysis was performed and receiver operating characteristic (ROC) analysis was used to determine the performance and the sensitivity/specificity for individual follicular fluids for which the outcome was clearly defined as either no delivery $(n=104)$ or definite delivery $(n=13)$. Covariate factors known to influence the IVF outcome such as age, number of previous failed IVF attempts and embryo score were systematically integrated in each analysis, in order to exclude possible bias in the interpretation. A $p$-value $>0.1$ was used as a criterion for exclusion according to the literature on multivariate prognostic modeling. The following thresholds were used to interpret area under the ROC curve $\left(\mathrm{AUC}_{\mathrm{ROC}}\right)$ : 0.9-1, perfect separation; 0.8-0.9, excellent discrimination; 0.7-0.8, acceptable discrimination; 0.6-0.7, poor discrimination; 0.5-0.6, no discrimination (MedCalc for Windows, version 9.2.0.0 software, MedCalcSoftware, Mariakerke, Belgium). The $\mathrm{AU}_{\mathrm{roc}}$ methodology also allowed us to define the threshold of optimal sensitivity/specificity according to each method of quantification in regard to the delivery outcome. We then compared the implantation rate of the entire cohort of embryos transferred $(n=139)$ according to their predefined category of G-CSF concentrations by ANOVA test. A p-value of $<0.05$ was considered as significant. 
Table 1 Patient profile and response to ovarian stimulation in the three predefined groups of embryos.

\begin{tabular}{|c|c|c|c|c|}
\hline Parameter & $\begin{array}{c}\text { No implantation } \\
{[\mathrm{IR}=0]}\end{array}$ & $\begin{array}{c}\text { Definite implantation } \\
{[\mathrm{IR}=1]}\end{array}$ & $\begin{array}{c}\text { Likely implantation } \\
{[0<\mathrm{IR}<1]}\end{array}$ & $p$-Value ${ }^{\mathrm{a}}$ \\
\hline Number of patients & 62 & 7 & 15 & \\
\hline Age (years) & 32.35 & 30.4 & 29.6 & 0.05 \\
\hline $\begin{array}{l}\text { Protocol of ovarian hyperstimulation \% } \\
\text { antagonist/\%agonist }\end{array}$ & $16 / 84$ & $14 / 86$ & $16 / 84$ & NS \\
\hline $\begin{array}{l}\text { Oestradiol in serum on day HCG administration } \\
(\mathrm{pg} / \mathrm{ml})^{\mathrm{b}}\end{array}$ & 2485 & 1898 & 2350 & 0.4 \\
\hline Number of oocytes collected ${ }^{b}$ & 8.56 & 8.7 & 8.86 & 0.96 \\
\hline Number of embryos obtained at day $2^{\mathrm{b}}$ & 5 & 4 & 4.6 & 0.4 \\
\hline Mean number of embryos transferred on day $2^{b}$ & 2 & 1.85 & 1.9 & 0.4 \\
\hline$\%$ of twins & 0 & $86(6 / 7)$ & $0(0 / 22)$ & 0.0001 \\
\hline $\begin{array}{l}\text { FF analysed by both multiplexed microbead } \\
\text { assays and ELISA }\end{array}$ & 104 & 13 & 22 & \\
\hline
\end{tabular}

FF, follicular fluids.

a Data were analysed by ANOVA.

${ }^{\mathrm{b}}$ Data are mean values.

\section{Results}

\subsection{ICSI results, pregnancy rates and delivery rates}

The patient profiles, response to stimulation and IVF/ICSI outcome are detailed in Table 1 for the three predefined categories of outcome (no implantation, definite implantation and likely implantation). No significant difference was observed between the groups of embryos which implanted or not for the age of patients, their respective response to ovarian stimulation, number of oocytes collected or number of embryos transferred. Overall, the mean implantation and delivery rates were $17.3 \%$ per embryo. No miscarriage or non-progressing biochemical pregnancies were observed in the present cohort (28 gestational sacs for 162 transferred embryos). Per patient, the mean clinical pregnancy and live birth rates were 25\% (21/84), and the multiple pregnancy rate was $33 \%(7 / 21)$.

\subsection{The cytokine/chemokine/growth factor content of follicular fluid samples}

In the Bio-Rad multiplexed microbead assay IL-1R $\alpha$, IL-4, IL-6, IL-8, IL-12, IL-13, G-CSF, VEGF, MCP-1, and eotaxin were detected in all follicular fluid samples, while IL-1 $\alpha$, IL-5, IL-7 and IL-17 were detected in none or less than $5 \%$ of the samples. IL-15, PDGF, CCL-5, IFN7, IL-9, MIP-1 $\beta$, IL-10, GM-CSF, IP-10, IL-2, FGF, $\mathrm{TNF} \alpha, \mathrm{MIP} \alpha$ and CCL5 were detected in $95,88,82,81,81,78,62,59,58,38,36$ and 10\% of the follicular fluid samples respectively.

In the R\&D Systems microbead assay IL-1R $\alpha$, IL-4, IL-6, IL-8, G-CSF, MIP-1 $\beta$, CCL-5, MCP-1 and VEGF were detected in all follicular fluid samples, while IL-1 $\alpha$, IL-2 and IL-17 were detected in none or less than 5\% of the samples MIP-1 $\alpha$, TNF $\alpha$, IFN7, IL-10, GM-CSF and IL-5 were detected in 75, 68, 59, 30, 25 and $18 \%$ of the samples respectively.

In the standard G-CSF ELISA assay, G-CSF was detected in all samples.

In the multiplexed microbead assays from Bio-Rad and R\&D Systems, the means of follicular G-CSF were 18.7 and $21.6 \mathrm{pg} / \mathrm{ml}$ respectively, ranging from 0 to $37 \mathrm{pg} / \mathrm{ml}$ and 0 to $50.7 \mathrm{pg} / \mathrm{ml}$ respectively, with a normal distribution in both cases. In the ELISA, the mean value of follicular G-CSF was $130 \mathrm{pg} / \mathrm{ml}$, ranging from 47 to $313 \mathrm{pg} / \mathrm{ml}$, with an abnormal distribution (Agostino-Pearson test).

Follicular fluid G-CSF concentrations were significantly different between embryos, which led to implantation versus no implantation, in both of the multiplexed microbead assays from $\operatorname{Bio}-\operatorname{Rad}(p=0.01)$ and $\operatorname{R} \& D$ Systems $(p=0.04)$, but there was no difference between these groups detected by ELISA $(p=0.5)$ (Table 2$)$.

Surprisingly, most of the cytokines, chemokines and growth factors were not correlated with each other if assessed by the R\&D Systems or Bio-Rad multiplexed microbead assays, except for G-CSF, GM-CSF and 
VEGF values, which did show correlations. Furthermore, the follicular fluid G-CSF evaluated by the two multiplexed microbead assays from R\&D Systems and Bio-Rad were correlated $(r=0.38, p<0.001)$. The follicular fluid G-CSF evaluated by the multiplexed microbead assay and the ELISA from R\&D Systems were also correlated $(r=0.43, p<0.0001)$, but no correlation was observed between the G-CSF data evaluated by multiplexed microbead assay from Bio-Rad and the R\&D Systems ELISA.

Table 2 Concentrations of follicular fluid G-CSF in the three predefined groups of embryos accordingly to each of three methods of quantification.

\begin{tabular}{|c|c|c|c|c|}
\hline Parameter & $\begin{array}{l}\text { No implantation } \\
\quad[\mathrm{IR}=0]\end{array}$ & $\begin{array}{l}\text { Definite implantation } \\
\qquad[\mathrm{IR}=1]\end{array}$ & $\begin{array}{l}\text { Likely implantation } \\
\qquad[0<\mathrm{IR}<1]\end{array}$ & $p$-Value ${ }^{\mathrm{a}}$ \\
\hline $\begin{array}{l}\text { FF G-CSF (pg/ml) Multiplexed microbead assay } \\
\text { (Biorad) }^{\mathrm{b}}\end{array}$ & 17.6 & 22.9 & 21.3 & 0.01 \\
\hline $\begin{array}{l}\text { FF G-CSF }(\mathrm{pg} / \mathrm{ml}) \text { Multiplexed microbead assay } \\
(\mathrm{R} \& D \text { Systems) }\end{array}$ & 20.6 & 28.9 & 21.9 & 0.04 \\
\hline FF G-GSF (pg/ml) ELISA (R\&D Systems) ${ }^{b}$ & 127 & 140 & 135 & 0.5 \\
\hline
\end{tabular}

FF, follicular fluids.

${ }^{a}$ Data were analysed by ANOVA, $p$-values for difference between no implantation and definite implantation groups are given.

${ }^{\mathrm{b}}$ Data are mean values.

\subsection{Follicular fluid G-CSF detection by multiplexed microbead assay is predictive for implantation and delivery} rates

To discriminate between oocytes that lead to a successful birth and those that do not, follicular fluid G-CSF was the only variable retained in the multivariate logistic model as significant. The $\mathrm{Au}_{\mathrm{roc}}$ was 0.77 [0.66-0.82] $(p=$ $0.003)$ for follicular fluid G-CSF evaluated by Bio-Rad multiplexed microbead assay and $0.75[0.68-0.84](p=$ 0.004 ) for follicular fluid G-CSF evaluated by R\&D Systems multiplexed microbead assay (Fig. 1). The optimal threshold according to the ROC curves for follicular fluid G-CSF was $20.54 \mathrm{pg} / \mathrm{ml}$, with a sensitivity of $81.8 \%$ for a specificity of $62.3 \%$ for the Bio-Rad multiplexed microbead assay, and $24.9 \mathrm{pg} / \mathrm{ml}$ with a sensitivity of $81.8 \%$ for a specificity of $68.6 \%$ for the R\&D Systems multiplexed microbead assay.

In contrast, for follicular fluid G-CSF quantified by ELISA, none of the data included were retained in the multivariate model. The $\mathrm{Au}_{\mathrm{roc}}$ for follicular fluid G-CSF evaluated by ELISA was not significant $\left(\mathrm{Au}_{\mathrm{roc}}=0.61\right.$ $[0.52-0.70], p=0.84)$ and not able to discriminate between oocytes, which will lead to delivery and those that did not.

Fig. 1. Area under the ROC curve for follicular G-CSF assessed by multiplexed bead-based assay purchased from Bio-Rad and R\&D Systems, and standard ELISA.

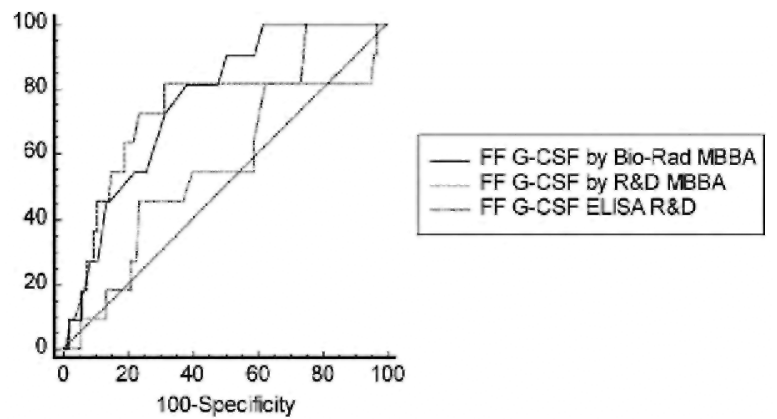

Neither age, serum oestradiol level, the treatment day on which ovulation was triggered, nor the embryo score were retained in any models using multivariate logistic regression. None of the factors evaluated by multiplexed microbead assay were different as a function of embryo morphology, fragmentation or early cleavage. The 
embryo morphology appeared as an independent factor in regard to follicular fluid G-CSF concentration (data not shown).

However, we did observe a decrease in follicular fluid G-CSF content with each of the three methods of detection as a function of the oestradiol response to the hormonal stimulation. Between low, normal and high responders, follicular fluid G-CSF concentrations decreased from 25.3 to $21.6 \mathrm{pg} / \mathrm{ml}$ and $18.0 \mathrm{pg} / \mathrm{ml}$ with the R\&D Systems multiplexed microbead assay, from 22.5 to $17.8 \mathrm{pg} / \mathrm{ml}$ and $19.0 \mathrm{pg} / \mathrm{ml}$ with the Bio-Rad multiplexed microbead assay, and from 158 to $128 \mathrm{pg} / \mathrm{ml}$ and $109 \mathrm{pg} / \mathrm{ml}$ with the G-CSF ELISA. The decrease was significant between low and normal responders with the Bio-Rad multiplexed microbead assay $(p=0.04)$ and the ELISA $(p=0.002)$. This suggests that an excessive ovarian response could induce a downregulation of preovulatory follicular fluid G-CSF synthesis.

According to the optimal threshold of G-CSF concentrations defined by the ROC curve (optimal sensitivity/specificity) as predicting the potential for live birth, we compared the \% of delivery among all the embryo cohorts (Table 3). Only follicular fluid G-CSF quantified by multiplexed microbead assays from either Bio-Rad or R\&D Systems was significant in regard to the delivery rates.

Table 3 Comparison of delivery rate as a function of each method of quantification of follicular fluid G-CSF, with the optimal threshold of concentration defined by the ROC curves.

\begin{tabular}{lcccccc}
\hline & $\begin{array}{c}\text { Multiplexed microbead assay } \\
\text { (R\&D Systems) }\end{array}$ & \multicolumn{2}{c}{$\begin{array}{c}\text { Multiplexed microbead assay } \\
\text { (Bio-Rad) }\end{array}$} & \multicolumn{2}{c}{ ELISA } \\
\hline FF G-CSF (pg/ml) & $<24.9$ & $>24.9$ & $<20.54$ & $>20.54$ & $<149$ & $>149$ \\
Number of embryos & 92 & 47 & 79 & 60 & 98 & 41 \\
Delivery rate (\%) & 11.0 & 24.4 & 9.6 & 24.2 & 13.7 & 20.7 \\
$p$-Value & 0.01 & & 0.006 & & 0.11 & \\
\hline
\end{tabular}

For all three methods, discriminating thresholds for follicular fluid G-CSF concentration were established from the AUroc curve and defined by the optimal sensitivity/specificity. The entire cohort of embryos observed was then classified as a function of follicular fluid G-CSF concentrations and the corresponding delivery rate values are compared.

$\mathrm{FF}$, follicular fluid.

${ }^{a}$ Data were analysed by ANOVA, $p$-values for difference between delivery rates between embryos classified above and below thresholds are given.

\subsection{Comparison of follicular fluid G-CSF quantification by multiplex and single-plex G-CSF microbead assay}

In order to evaluate if multiplexing cytokine detection could have an impact on a single cytokine measurement in follicular fluid, we compared the quantification of follicular fluid G-CSF by multiplex versus single-plex microbead assay purchased from the same company (Bio-Rad) for a separate cohort of 25 undiluted follicular fluids collected in 2006. Follicular fluid G-CSF were highly and significantly correlated $(r=0.72, p<0.0001)$ suggesting that detection of follicular fluid G-CSF is not influenced by the multiplex detection platform (Fig. 2a). Compared with the recently collected follicular fluid samples, we observed an approximate 10-fold decrease in follicular fluid G-CSF concentrations in the 25 follicular fluids that were collected in 2006 and had undergone storage, transport and a few cycles of freezing and thawing before the microbead assay.

\subsection{Effect of follicular fluid dilution on follicular fluid G-CSF quantification by bead-based assay}

To document if compounds could interact and influence subsequent G-CSF quantification, we analysed the effect of follicular fluid dilution on subsequent quantification of G-CSF for two pooled follicular fluid samples previously documented to have a low versus high concentration in single-plex microbead assay. Follicular fluid dilution acted to influence the amount of follicular fluid G-CSF measured, and increased the detectability of follicular fluid G-CSF detected by single-plex microbead assay (Fig. 3). This was likely to occur through decreasing the concentration in samples of compounds known to bind to cytokines, such as albumin, alpha-2 microglobulin and autoantibodies, 
3.6. Comparison of G-CSF quantification by single-plex G-CSF microbead assay and G-CSF flow cytometric assay

To evaluate if differences observed in function of each methodology applied was related mainly to antibodies and/or to the principles of detection, we compared the follicular fluid G-CSF concentrations observed with single-plex G-CSF microbead assay versus G-CSF Cytometric Bead Array flow cytometry assay. Twenty-seven diluted follicular fluids (1/10) with no previous thawing were simultaneously analysed. Follicular fluid G-CSF concentrations evaluated by single-plex microbead assay and the Cytometric Bead Array were highly correlated $(r=0.95, p<0.0001)$ (Fig. 2b).

Fig. 2. (a) Correlation between follicular fluid G-CSF evaluated by multiplex versus single-plex G-CSF microbead assay (both Bio-Rad) on 25 previously thawed, undiluted follicular fluid samples $(r=0.72, p<$ 0.0001). (b) Correlation between follicular fluid G-CSF evaluated by single-plex G-CSF microbead based assay versus Cytometric Bead Array on 27 diluted (1:10) follicular fluids, without prior thawing $(r=0.95, p<$ $0.0001)$.

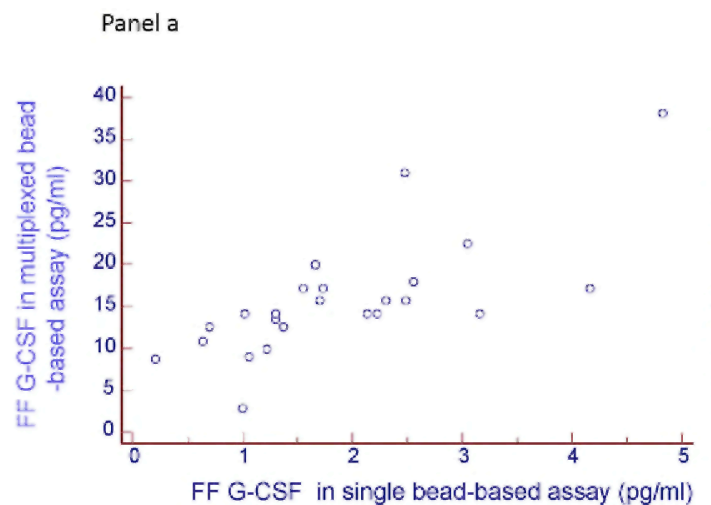

Panel b

FF G-CSF in single bead-based assay (pg/ml)

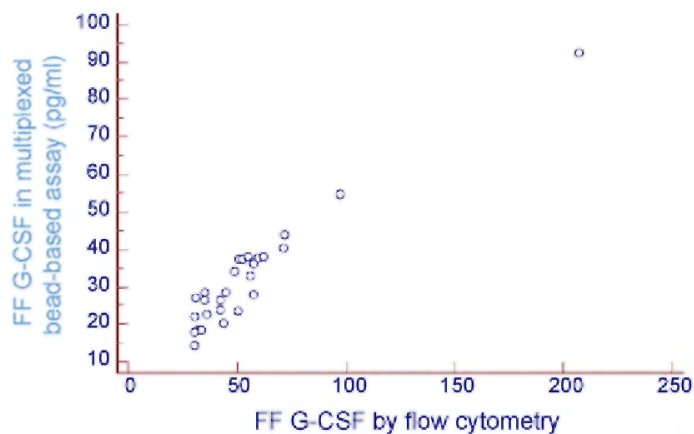

Fig. 3. Effect of follicular fluid dilutionon subsequent follicular fluid G-CSF quantification by single-plex microbead assay.

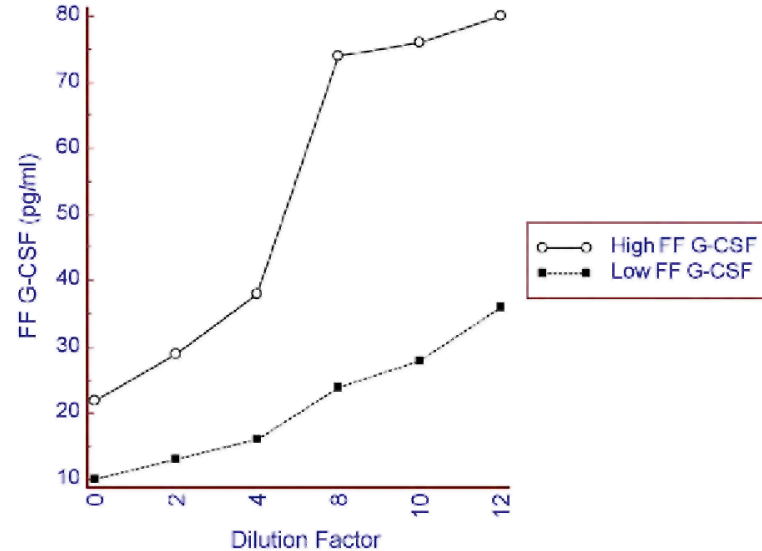

\section{Discussion}

This study confirmed previous findings that G-CSF evaluation in individual follicular fluids is a non-invasive biomarker of oocyte competence, able to predict which oocytes will yield embryos capable of effective implantation. The current study highlights the importance of the methodology utilised to quantify the follicular fluid G-CSF. Indeed, microbead assays but not a standard commercial ELISA yielded values of follicular G-CSF that allow discrimination of oocytes in regard to their individual competence for future implantation. 
The different sensitivities in the detection of the different cytokines of the three assays might be due to many reasons, such as the affinity or avidity of the various antibodies used, the incubation buffer, as well as variations in the manufacturers' recommended protocols. The clone of monoclonal antibodies used for detection and reporting are pivotal in obtaining identical results between assays (Elshal and McCoy, 2006) and are likely to explain the differences between the two microbead assay methods. Indeed, almost none of the analytes evaluated by either the R\&D Systems or the Bio-Rad microbead assays were correlated. Factors implicated as causing differences are compounds known to bind to cytokines, such as albumin, alpha-2 microglobulin and autoantibodies (Aziz et al., 1999; de Jager et al., 2005). Consistent with this, we were able to observe a clear effect of dilution on subsequent quantification of follicular fluid G-CSF. Furthermore, there are substantial differences between microbead assay and ELISA platforms. Microbead assays use fluorescence as the reporter system whereas ELISA uses enzyme amplification of a colorometric substrate. Microbead assays capture ligands onto spherical beads in suspension while ELISA relies upon the well surface in a 96-well plate. The high correlation of follicular fluid G-CSF content seen between the microbead assay and flow cytometry suggests that ligand capture onto spherical beads, which occurs in both these methods, is an essential element in increasing assay sensitivity.

The microbead assay technology has been developed for its advantages of time and cost saving due to the capabilities of multi-analysis in volumes as small as $50 \mu \mathrm{l}$. In the present application, we do not require a high throughput analysis of numerous analytes and do not have limited sample volume (the mean volume of individual follicular fluid samples is $2 \mathrm{ml}$ ). The cost related to purchase and maintenance of the Luminex system as well as the purchase of disposable kits is clearly the limitation for widespread uptake of this diagnostic assay into routine clinical application.

Most authors who have compared microbead assays to ELISAs report high correlations (especially when kits originate from the same manufacturer), higher or lower sensitivities in various ranges of the standard curve, and a lack of agreement between the computed absolute protein values, as observed here (Dupont et al., 2005; Elshal and McCoy, 2006; Pickering et al., 2002; Young et al., 2008). A key concern is the possibility that multiplexing itself results in anomalies in the quantification of some of the analytes. The high correlation we observed between multiplex and single-plex microbead assay data indicates that multiplexing is not necessary for follicular fluid G-CSF quantification in follicular fluid.

To apply this test to a routine clinical setting, standard operating procedures will need to be fully documented and validated through inter-laboratory comparisons. The importance of sample collection, processing and storage in affecting the validity of the measurement and levels of cytokines in biological fluids has been demonstrated (Aziz et al., 1999). Dilution of samples may be suitable for optimal quantification, especially for low concentrations. We also observed that in comparison with previous studies, the detection threshold depends on the lot number of the microbead assay, suggesting that uniformity in the standards for quantitative assays is required. For example, appropriately stored aliquots of pooled follicular fluids might be used as inter-run calibrators.

To conclude, follicular fluid G-CSF appears to be a non-invasive biomarker of human oocyte developmental competence. Since the comparison of absolute values is crucial for effective consequences in the choice of embryos, designing a quick, routine test, which displays an optimal sensitivity and a high specificity is now an absolute requirement.

\section{Conflict of interest}

The authors have applied for a PCT patent of application (PCT/EP2007/057430) July, 2007.

\section{Acknowledgments}

For the collection of the individual follicular fluid samples, we thank Marc Bailly and Juliette Guibert. For the handling, storage and transport of the individual follicular fluid samples, we thank Marie Petitbarat and Sylvie Dubanchet. We thank Ibrahim Hammoud, Marianne Bergère, Jacqueline Selva and Francois Vialard and the technicians (Patrick Cavelot, Vincent Delabroye, Virginie Le Bail) from the Reproductive Medicine Unit of Poissy-St Germain en Laye for the extra work they kindly performed. We thank Corinne Morvan for conducting ELISAs, Marie-Rose Pignon for the single-plex bead-based assay and flow cytometry experiments and Sandra Ormenese from the GIGA-Flow Cytometry Platform. We also thank all the researchers of the European EMBIC networks, who helped in these achievements and in the construction of future directions and understanding. 


\section{References}

Aziz, N., Nishanian, P., Mitsuyasu, R., Detels, R., Fahey, J.L., 1999. Variables that affect assays for plasma cytokines and soluble activation markers. Clin. Diagn. Lab. Immunol. 6, 89-95.

de Jager, W., Prakken, B.J., Bijlsma, J.W., Kuis, W., Rijkers, G.T., 2005. Improved multiplex immunoassay performance in human plasma and synovial fluid following removal of interfering heterophilic antibodies. J. Immunol. Methods 300, 124-135.

Dupont, N.C., Wang, K., Wadhwa, P.D., Culhane, J.F., Nelson, E.L., 2005. Validation and comparison of luminex multiplex cytokine analysis kits with ELISA: determinations of a panel of nine cytokines in clinical sample culture supernatants. J. Reprod. Immunol. 66, 175191.

Elshal, M.F., McCoy, J.P., 2006. Multiplex bead array assays: performance evaluation and comparison of sensitivity to ELISA. Methods 38, $317-323$.

Guerif, F., Le Gouge, A., Giraudeau, B., Poindron, J., Bidault, R., Gasnier, O., et al., 2007. Limited value of morphological assessment at days 1 and 2 to predict blastocyst development potential: a prospective study based on 4042 embryos. Hum. Reprod. 22, $1973-1981$.

Lédée, N., Lombroso, R., Lombardelli, L., Selva, J., Dubanchet, S., Chaouat, G., et al., 2008. Cytokines and chemokines in follicular fluids and potential of the corresponding embryo: the role of granulocyte colony-stimulating factor. Hum Reprod 23, 2001-2009.

Lédée N., Frydman R., Osipova A., Taieb J., Gallot V., Lombardelli L., Logiodice F., Petitbarat M., Fanchin R., Chaouat G., AchourFrydman N., Piccinni M.P. Levels of follicular G-CSF and interleukin-15 appear as noninvasive biomarkers of subsequent successful birth in modified natural in vitro fertilization/intracytoplasmic sperm injection cycles. http://www.ncbi.nlm.nih.gov/pubmed/20400073 Fertil Steril. 2010 Apr 16. [Epub ahead of print] PMID: 20400073.

Patrizio, P., Sakkas, D., 2009. From oocyte to baby: a clinical evaluation of the biological efficiency of in vitro fertilization. Fertil. Steril. 91 , $1061-1066$

Pickering, J.W., Martins, T.B., Schroder, M.C., Hill, H.R., 2002. Comparison of a multiplex flow cytometric assay with enzyme-linked immunosorbent assay for auantitation of antibodies to tetanus, diphtheria, and Haemophilus influenzae Type b. Clin. Diagn. Lab. Immunol. $9,872-876$

Salmassi, A., Schmutzler, A.G., Huang, L., Hedderich, J., Jonat, W., Mettler, L., 2004. Detection of granulocyte colony-stimulating factor and its receptor in human follicular luteinized granulosa cells. Fertil. Steril. 81 (Suppl. 1), 786-791.

Salmassi, A., Schmutzler, A.G., Schaefer, S., Koch, K., Hedderich, J.,Jonat, W., et al., 2005. Is granulocyte colony-stimulating factor level predictive for human IVF outcome? Hum. Reprod. 20, 2434-2440.

Steer, C.V., Mills, C.L., Tan, S.L, Campbell, S., Edwards, R.G., 1992. The cumulative embryo score: a predictive embryo scoring technique to select the optimal number of embryos to transfer in an in-vitro fertilization and embryo transfer programme. Hum. Reprod. $7,117-119$.

Yanagi, K., Makinoda, S., Fujii, R., Miyazaki, S., Fujita, S., Tomizawa, H., et al., 2002. Cyclic changes of granulocyte colony-stimulating factor(G-CSF) mRNA in the human follicle during the normal menstrual cycle and immunolocalization of G-CSF protein. Hum. Reprod. 17, $3046-3052$

Young, S.H., Antonini, J.M., Roberts, J.R., Erdely, A.D., Zeidler-Erdely, P.C., 2008. Performance evaluation of cytometric bead assays for the measurement of lung cytokines in two rodent models. J. Immunol. Methods 331, 59-68. 